\title{
Collecting Potential Optimisations
}

\author{
Nancy Mazur, Gerda Janssens, and Wim Vanhoof \\ Department of Computer Science, K.U.Leuven \\ Celestijnenlaan 200A, B-3001 Heverlee, Belgium \\ $\{$ nancy, gerda, wimvh\}@cs. kuleuven.ac.be
}

\section{Introduction and Motivation}

Most program analyses and optimisations face the problem of controlling polyvariance, i.e. deciding in how many ways a single program entity is analysed and/or annotated for optimisation. In the context of logic programming, an analysis creates a number of versions for each original predicate, where each such version corresponds with a particular use or optimisation of the predicate. There is a trade-off between the number of versions (the size of the program) and the optimality of the resulting program.

The problem can be tackled in different ways. The classic approach is to analyse the program in a top-down fashion, starting from an initial call, and to create a different version of a predicate for each description of a call that occurs during the analysis. To counter the creation of too many versions, a pruning phase can be introduced, like in [3]. A disadvantage of such a calldependent approach is that the program typically needs to be reanalysed for each newly encountered use of a predicate (as the underlying analysis usually only guarantees that the optimisations are safe for a restricted number of uses of the predicate). Moreover, if the analysis limits the number of versions that it creates, it mainly achieves this by widening or abstraction of the set of encountered call descriptions without taking into account the optimisations induced by these call descriptions. Another typical approach to version control is the one we followed in the context of compile-time garbage collection, where we generate at most two versions of each predicate: a non-optimised version that is always safe to call, and a fully optimised version that can only be used if the caller of the predicate meets the (often harsh) conditions that guarantee that all the optimisations can safely be performed. Although there is no risk for code explosion, a lot of intermediate opportunities for optimisations are missed.

The underlying problem is that most of the approaches lack an adequate mechanism for collecting and comparing the possible optimisations before the actual versions are created. In this work we propose an optimisation derivation system that derives the explicit relations that exist between a particular optimisation opportunity within a predicate and the requirements on the call that allow the optimisation to be safely performed. This information allows to (automatically) reason about the versions that are interesting to generate (e.g. how many and which optimisations are induced by a particular call description); it can be interesting feedback to the programmer (e.g. why can some specific atom 
not be optimised?); and finally, such a system can be seen as a step towards conceptually separating the analysis of a program from the generation of the versions, opening the field for better theoretical insights into the latter problem.

\section{Optimisation Derivation Framework}

We assume that the user program comes pre-annotated with goal-independent information at each atom of interest. This information is expressed in terms of an abstract domain $\mathcal{A}$ upon which the intended optimisations for these atoms can be decided. We require that $\mathcal{A}$ has a (monotone) combination operator $\otimes$ : $\mathcal{A} \times \mathcal{A} \rightarrow \mathcal{A}$ which is used to safely approximate goal-dependent information given a description of a specific call and a goal-independent annotation.

The atoms of interest consist of base atoms - atoms for which the optimisation criteria are predefined, and call atoms - predicate calls through which optimisation criteria are propagated. Let $o_{a}$ be a function that, when applied to a goal-dependent abstract description for the atom $a$, returns true if the intended optimisation for $a$ is safe for that description, and false otherwise.

Consider the predicate definition: $p \leftarrow \ldots a, \ldots$ Let $a$ be an atom of interest, and let $\iota_{a}$ be the goal-independent annotation of $a$. Let $\delta_{p}$ be a description of the call to $p$, then we can verify the optimisation of $a$ by computing $o_{a}\left(\iota_{a} \otimes \delta_{p}\right)$. Our idea is to use the above formula to find such a "most general" $\delta_{p}$. This can be done by computing the generalised pseudo-complement of $\iota_{a}$ w.r.t. $\otimes$ (much like the pseudo-complement is used in the backwards propagation of call patterns [1]). Using the pseudo-complement, a new function $o_{p}$ can be generated with which the optimisation of $a$ within calls to $p$ can be checked by checking the goal-dependent abstract descriptions of calls to $p$. If each predicate contains only one atom of interest, then the propagation of these functions up the call graph is straightforward. If $p$ contains several atoms of interest, their optimisation functions need to be combined in order to propagate them up the call graph. There are a number of ways to combine these functions: e.g. by using the least upper bound or the greatest lower bound, or by collecting them as sets. The exact choice has an effect on the information resulting from the analysis: either the system derives necessary conditions or sufficient conditions. Further details are discussed in [2].

\section{References}

1. A. King and L. Lu. A Backward Analysis for Constraint Logic Programs. Theory and Practice of Logic Programming, 2(4-5):517-547, July-September 2002.

2. N. Mazur, G. Janssens, and W. Vanhoof. Collecting Potential Optimisations. Report CW 357, Department of Computer Science, K.U.Leuven, Leuven, Belgium, February 2003.

3. G. Puebla and M. Hermenegildo. Abstract Multiple Specialization and its Application to Program Parallelization. Journal of Logic Programming. Special Issue on Synthesis, Transformation and Analysis of Logic Programs, 41(2\&3):279-316, November 1999. 